\title{
ARTICLE
}

\section{Study of anatomical feature of in vitro and ex vitro regeneration plant of Sophora Alopecuroides $l$.}

\author{
Tsolmon M. ${ }^{1}$, Bayarmaa B. ${ }^{2}$ and Oyunbileg Yu. ${ }^{1 *}$ \\ ${ }^{1}$ Plant Biotechnology Laboratory, Institute of Biology, \\ Mongolian Academy of Sciences, Ulaanbaatar, Mongolia \\ ${ }^{2}$ Anatomy and Ecophysiology Laboratory, Botanyic garden and research institute, \\ Mongolian Academy of Sciences, Ulaanbaatar, Mongolia
}

ARTICLE INFO: Received: 20 May, 2019; Accepted: $30 \mathrm{Jul}, 2019$

\begin{abstract}
The success of shoot and rooting from the nodal shoot segments of Sophora alopecuroides L. were induced in vitro condition. However, transferring and acclimatizing the plantlets to under soil or ex vitro condition were difficult. This study investigated plant survival with anatomical changes in plantlets while transferring from in vitro to ex vitro conditions to investigate vascular cylinder variations.

The ex vitro rooting of the in vitro regenerated shoots, after having been treated with $500 \mathrm{mg} / \mathrm{LIBA}$, showed a success rate of 80 per cent. These plantlets were rooted and acclimatized simultaneously in ex vitro condition.
\end{abstract}

Keywords: Sophora alopecuroides; regeneration; ex vitro rooting; anatomy; central cylinder;

\section{INTRODUCTION}

Sophora alopecuroides Linn., a perennial shrub of Fabaceae family has been used in Mongolian traditional medicine to treat fever, bacterial infection, heart disease, and rheumatism [7]. This plant made up for more than 25 prescriptions of Mongolian traditional medicine, such as Mana-4, 10, 15, Norov-7, Marchin-13, Lider-5, 7, Arjutan, Buurun shosh-7, Bariav-17, Banlag-3, Jamba-6, Jonlan-5, Tanchin-10, Tsulkhir-4, Agar-15, 35, Ar Ür-14, 21, Balchin-23, Boigar-10, 18, Giban-13, and Khiin Gurgum-7 [2]. Sophora is a genus encompassing about 45 species, and only two of them have been so far recorded in Mongolia.

The population of this genus is declining [10] due to overharvesting of the roots for medicinal purposes. This species has been assessed as endangered owing to poor regeneration in nature and very limited geographic distribution in Mongolia [6]. Also, the germination rate of the seed is low, less than $50 \%$, according to previous reports [18]. In order to protect or save the natural resources for this species, it is necessary to propagate them in vitro condition. 
As indicated in the results of other research groups, direct shoot regeneration from nodal segments has been implemented successfully for this species, however, acclimatization was the biggest challenge $[1,8,17,18]$.

Tissue culture system is useful for rapid multiplication of improved varieties and for production of disease-free and goodquality planting materials [9]. The plant growth regulators were tested and conditions were optimized for efficient shoot and root development in $S$. alopecuroides during present investigation. Nevertheless, there are few

\section{MATERIALS AND METHODS}

\section{Collection of plant material and preparation of explants}

The seeds of Sophora alopecuroides were collected from a place called Ekhiin Gol in Bayankhongor aimag in the south-west of Mongolia. The seeds were treated with $70 \%$ ethanol for 3 minutes, followed by surface sterilization in $1 \% \mathrm{NaOCl}$ with $2-3$ drops of Tween-20 for 10 minutes and then rinsed with sterilized distilled water five times under aseptic conditions. The germinated seedlings were cut into 3-4 cm with 1-2 nodes.

\section{Establishment of culture}

Nodal segments were inoculated on agar gelled $(0.8 \%$ agar) MS medium (Murashige and Skoog 1962) supplemented with various combinations of 6-benzylaminopurine (BAP) $(0.5-2.0 \mathrm{mg} / \mathrm{L})$ and $\alpha$-Naphthalene acetic acid (NAA) $(0.2-0.5 \mathrm{mg} / \mathrm{L})$ or only indole-3-butyric acid (IBA) $(0.5-2.0 \mathrm{mg} / \mathrm{L})$ for shoot induction. Culture condition was $16: 8 \mathrm{~h}$ light:dark of photo period at $25 \pm 2^{\circ} \mathrm{C}$ temperature.

\section{Multiplication of shoots in cultures}

The shoots were cut into $2-4 \mathrm{~cm}$ long segments (each with at least 1-2 nodes) and subcultured on fresh MS medium with a 3 to 4 week interval. The culture medium used for shoot multiplication was incorporated with various concentrations and combinations 0.5-2.0 $\mathrm{mg} / \mathrm{L}$ 6-benzylaminopurine (BAP), indole-3-butyric acid (IBA) and 0.2-0.5 mg/L $\alpha$-Naphthalene acetic acid (NAA). anatomical studies showing how and where the adventitious organs are formed during ex vitro adaptation. Such information could be useful in understanding the adaptive mechanisms of many plants during the transition steps from in vitro to the soil [11]. In this study, we report the direct root regeneration protocol in the soil which could skip some stages of in vitro rooting and adaptation. The present study also aims to compare and evaluate anatomical developmental changes of roots in vitro, ex vitro and in vivo.

\section{Effect of auxins on rooting of proliferated shoots (in vitro)}

Well-developed shoots with 3-4 cm separated from bunch of shoots were transferred to half strength MS medium containing different concentrations of auxins (indole-3-butyric acid, IBA; indole-3 acetic acid, IAA and $\alpha$-Naphthalene acetic acid, NAA; $0.2-2.0 \mathrm{mg} / \mathrm{L}$ ). The cultures incubated under light for shoot induction with $16: 8 \mathrm{~h}$ light:dark photo period at $25 \pm 2^{\circ} \mathrm{C}$.

\section{Ex vitro root induction}

In order to skip some of additional steps for plantlet production, we conducted ex vitro root induction experiments. Healthy and tall shoots $(4-5 \mathrm{~cm})$ were separated one by one from the initial culture and the cut end of the shoots were dipped in 3 forms of auxin solution that were prepared in different concentration from 100 to $700 \mathrm{mg} / \mathrm{L}$ for $3 \mathrm{mins}$. The auxin solution consists of IAA, IBA and NAA. Finally, the auxin treated shoots were directly transferred to sterilized soil in bottles with different mixture (sand:soil:vermiculite), which were moistened regularly with one-fourth MS salts and maintained in ex vitro room with light for simultaneous rooting and hardening.

\section{Anatomical studies of root of $S$. alopecuroides}

For revealing the root maturing steps, we analysed the 2-month old plantlets (in vitro), 1 -month old plantlets rooted in ex vitro (shoots 
dipped in auxins solutions $(100-700 \mathrm{mg} / \mathrm{L})$ in ex vitro environment) and 1-year old plants adapted in greenhouse. The samples were fixed in solution with formalin, acetic acid, ethyl alcohol in the ratio of 1:1:18 for $24 \mathrm{~h}$. After the samples were cut by surgical blade, the sections were stained with $1 \%$ safranine for 4-8 $\mathrm{min}$. Stained sections were rinsed carefully in distilled water to remove excess stain. Photomicrograps were taken from the sections prepared in histological slides on the

\section{RESULTS AND DISCUSSION}

\section{Effects of phytohormones on multiplication} and establishment of cultures

In order to select the optimized phytohormone for shoot induction from the nodal segment, the MS medium (Murashige and Skoog 1962), supplemented with various concentrations and combinations of cytokinin (BAP) and auxins (such as IBA and NAA), were screened. The result revealed that the effect of only BAP containing variation (4.47.2 shoots) showed significant effect on shoot induction compared with that on medium with the combination of BAP together with NAA (2.2-3.7 shoots). However, abnormal shoots with leaf chlorosis and abscission developed on the medium having BAP alone. The number of shoots produced in this study was significantly greater than those achieved in the light microscope for 40x (Olympus ${ }^{\circledR}$ BX 41, Tokyo, Japan).

\section{Statistical analysis of the data}

The experiments were conducted with 20 explants for each treatment and repeated thrice. The results were subjected to analysis of variance and the significant differences among the mean values were estimated using Tukey's test at $\mathrm{p}<0.05$ using the Statistical Analysis System program (SAS, 2008).

earlier reports in S. flavescens [17, 18].

Tissue culture system has been successfully developed with nodal segment explant of S.flavescens through the application of many previous research results [1, 8, 15,17]. As was observed, the effectiveness of BAP over Kin for shoot initiation from nodal segment has been reported in Sophora's species. At the same time, it was reported that the combination of BAP and NAA was also useful for regeneration of Sophora toromiro [5]. Our results also indicated that only BAP-containing medium was appropriate as compared to the combination of BAP and NAA for proliferation. The method of multiplication of shoots by repetitive transfer of mother explants has been reported in many plants $[17,18]$. 


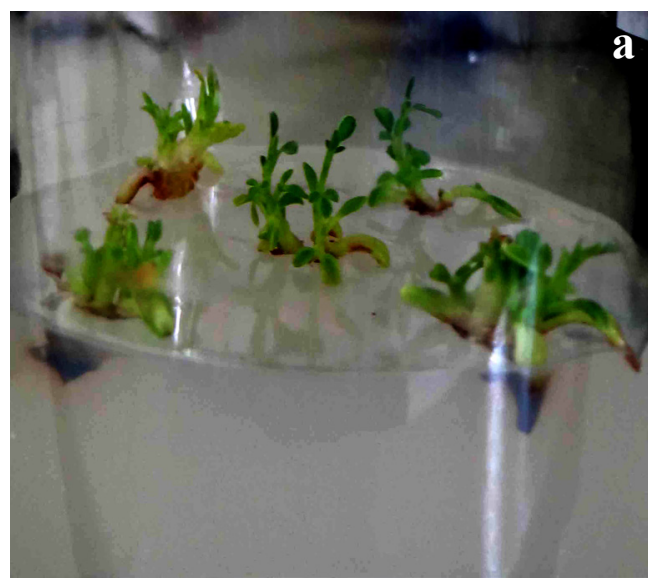

https://www.mongoliajol.info/index.php/PMAS
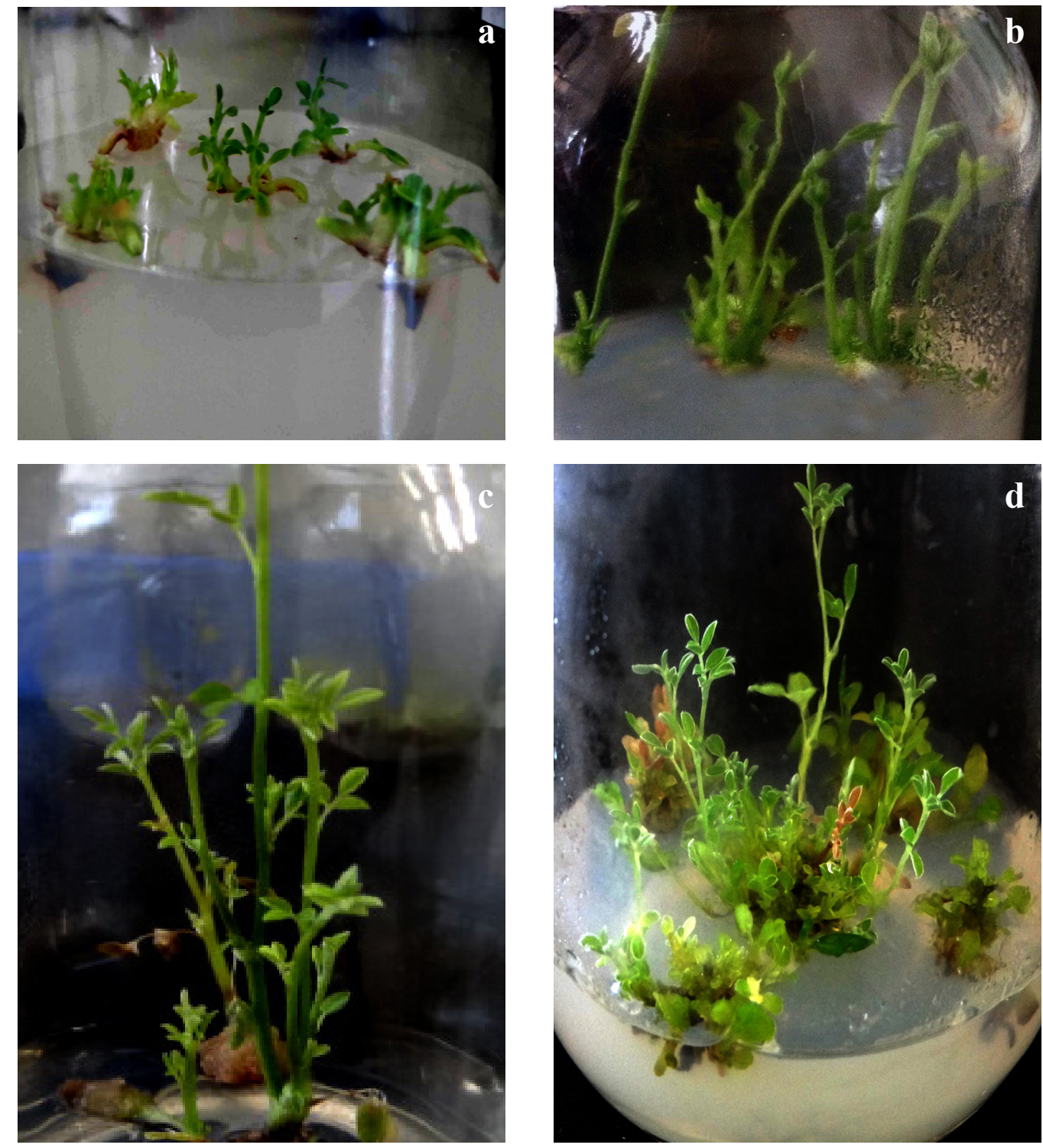

Figure 1. a: Bud breaking from the nodal region of explants.

$b$ : Multiple shoot induction.

c: Multiplication of shoots after 2 weeks.

$d$ : Shoots multiplication after 4 weeks. 


\section{Auxins and in vitro root induction}

The in vitro proliferated shoots were used for rooting experiments. For selection of a suitable medium of root induction, we tested half-strength MS medium having NAA, IAA and IBA $(0.2,1.0,2.0 \mathrm{mg} / \mathrm{L})$. Within the variations containing IBA, the highest results were indicated in the medium with $2.0 \mathrm{mg} / \mathrm{L}$, however, $34.8 \%$ of the explants could produce roots and the average root number was $3.9 \pm 0.4$ per explant and root length was about $1.9 \pm 0.1$.

IBA-derived auxin has strong impact on various aspects of root development, including regulation of root apical meristem size, root hair elongation, lateral root development, and the formation of adventitious roots [3]. Based on these theoretical information related with IBA, we tested the role of this hormone with 1.0-2.0 mg/L IBA concentration for rooting of proliferated shoots. Although the root induction rate was lower in all the variations containing $2.0 \mathrm{mg} / \mathrm{L} \mathrm{IBA}$, it highly promoted shoot proliferation with $10.6 \pm 0.5$ compared with BAP containing medium (Fig. 1).

MS medium supplemented with $2.0 \mathrm{mg} / \mathrm{L}$ IBA had more significant effect on shoot induction as compared to BAP containing variation. Depending on the plant species, some of them had highly synthesized cytokinin in them. Since enough cytokinin is accumulated in the plant naturally, it may require more auxins for root induction especially for this species. In our experiments, there were no root induction in all concentrations of NAA and semi- strength MS medium without hormone.

For $2.0 \mathrm{mg} / \mathrm{L} \quad$ IAA supplemented treatment, it was highly affective for rooting and percentage response was $71.5 \%$ with root number coming to $3.8 \pm 0.4$ and root length $1.2 \pm 0.1 \mathrm{~cm}$, indicating optimum for in vitro rooting from the cut ends of the shoots (Fig. 2a). The result from Cao and Li (2010) study supports our result of the strength of MS medium with IAA used for in vitro root induction.

\section{Ex vitro root induction and hardening of plantlets}

Experiments were carried out to achieve rooting and hardening simultaneously in ex vitro. Excellent results were obtained when the excised shoots were treated with IBA solutions for ex vitro root induction. Maximum response of rooting (100\%), number of roots per explant $(10.9 \pm 1.67)$ and root length $(1.57 \pm 0.12 \mathrm{~cm})$ were observed when treated with $500 \mathrm{mg} / \mathrm{L}$ concentration of IBA. The moderate rooting response was $7.26 \pm 0.33$ with roots per shoot recorded in all the variations containing IAA and the lowest results $(2.78 \pm 0.39$ roots per shoot) were observed in the control without any treatment. Since, our result is the first report on the ex vitro rooting of the shoots of $S$. alopecuroides, there weren't any previous reference for this species.

However, ex vitro root induction was successfully achieved in some other plants such as Ceropegia bulbosa [12], Leptadenia reticulata [13] and etc. This technique is time consuming and labor-intensive, which, however, could lead to the development of an effective in vitro plant propagation protocol for recalcitrant species on adaptation stage. The plantlets raised through ex vitro rooting methods are more suitable to tolerate the in vivo stresses [14].

The ex vitro rooted plantlets were hardened in ex vitro room condition. Plantlets in bottles with sterilized soil were transferred to soil containing plastic bottles without cap after 4-5 weeks (Fig. 2 d). Water spray on a daily basis was required for better survival rate during hardening of the plantlets in the greenhouse. The rate of survival at the time of hardening was maximum (80\%) during the present study. This result indicated that rooting in $e x$ vitro condition increased the survival rate by 2.4-fold compared with that of in vitro rooted plantlets. 


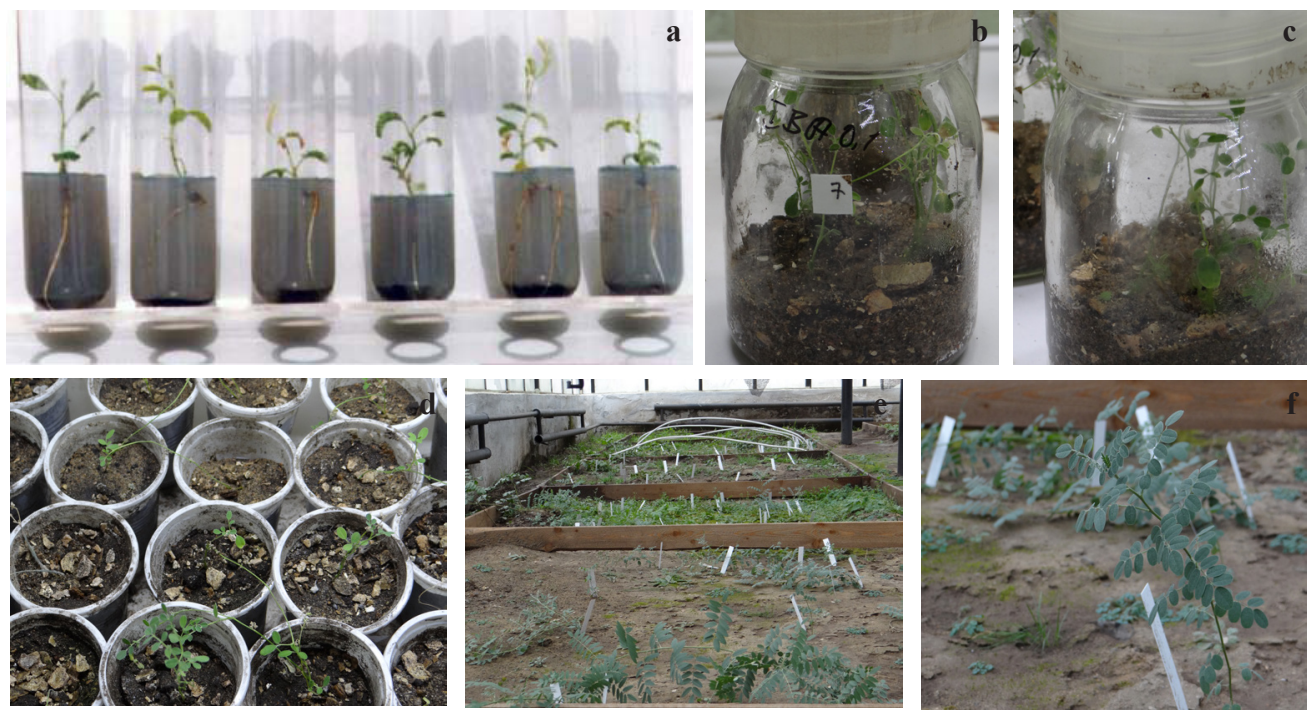

Figure. 2 a: Induction of in vitro roots from the cut ends of the shoots.

$b-d$ : Different stages in hardening of in vitro rooted plantlets.

e-f: Acclimatized plant in the greenhouse condition.

\section{Anatomical studies of root of}

\section{S. alopecuroides}

Normally, depending on the developmental stages of the root structure, 3 types of root structure were observed in the central cylinder (Fig.3) [16]. Abnormality during the in vitro propagation is due to special condition when plantlets grow in an environment with lowair circulation, high humidity and low-light intensity, and the root system develops poorly in plants.

As a result, the survival rate is highly reduced during the adaptation period. Thus, in order to show the root structure differences, samples from 1-year old plantlets adapted in green house, 2-month old in vitro rooted plantlets, and ex vitro rooted plantlets in different variations were used for anatomical study.

Epiblema, bark, pericycle and periderm layers were completely formed and the radialshaped xylem-phloem arrangement in the central cylinder was fully formed (Fig.4) for root structure of 1-year-old plants transferred in greenhouse.

Root structure of 2-month old plantlets grown in in vitro condition were characterized by epiblema, endodermis, pericyclic and peridermis cell and it also already started to develop central cylinder-like diarch form with primary phloem and xylem (Fig.5).

At the same time, all the variations of 30 days old plantlets, rooted in ex vitro condition, already obtained the diarch like structure and showed the fully developed central cylinder with primary phloem and xylem (Fig.6-8).

In contrast, as indicated in figure 9, root anatomical structure of 30-day old plantlets treated with $500 \mathrm{mg} / \mathrm{L}$ IBA showed that full set of primary and secondary epidermis were completely formed along with triarch-like structure of central cylinder with primary phloem and xylem. Sclerenchyma was also completely formed around them.

These results highlight the fact that the highest survival rate occurring in the plantlets rooted in ex vitro condition is related with the completely developed root system. The rapid development of vascular cylinder system with phloem and xylem were able to absorb water and nutrients from soil without any condition. The excellence of the root system, as mentioned by Gonçalves et al. (1998), is a key factor for the success of the acclimatization process. 


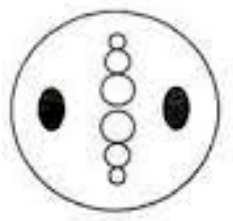

Diarch

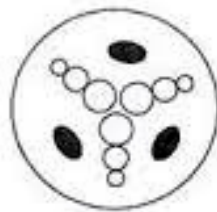

Triarch

Figure. 3: Diagram illustrating the different arrangements of the number of xylem groups in roots in crosssectional view
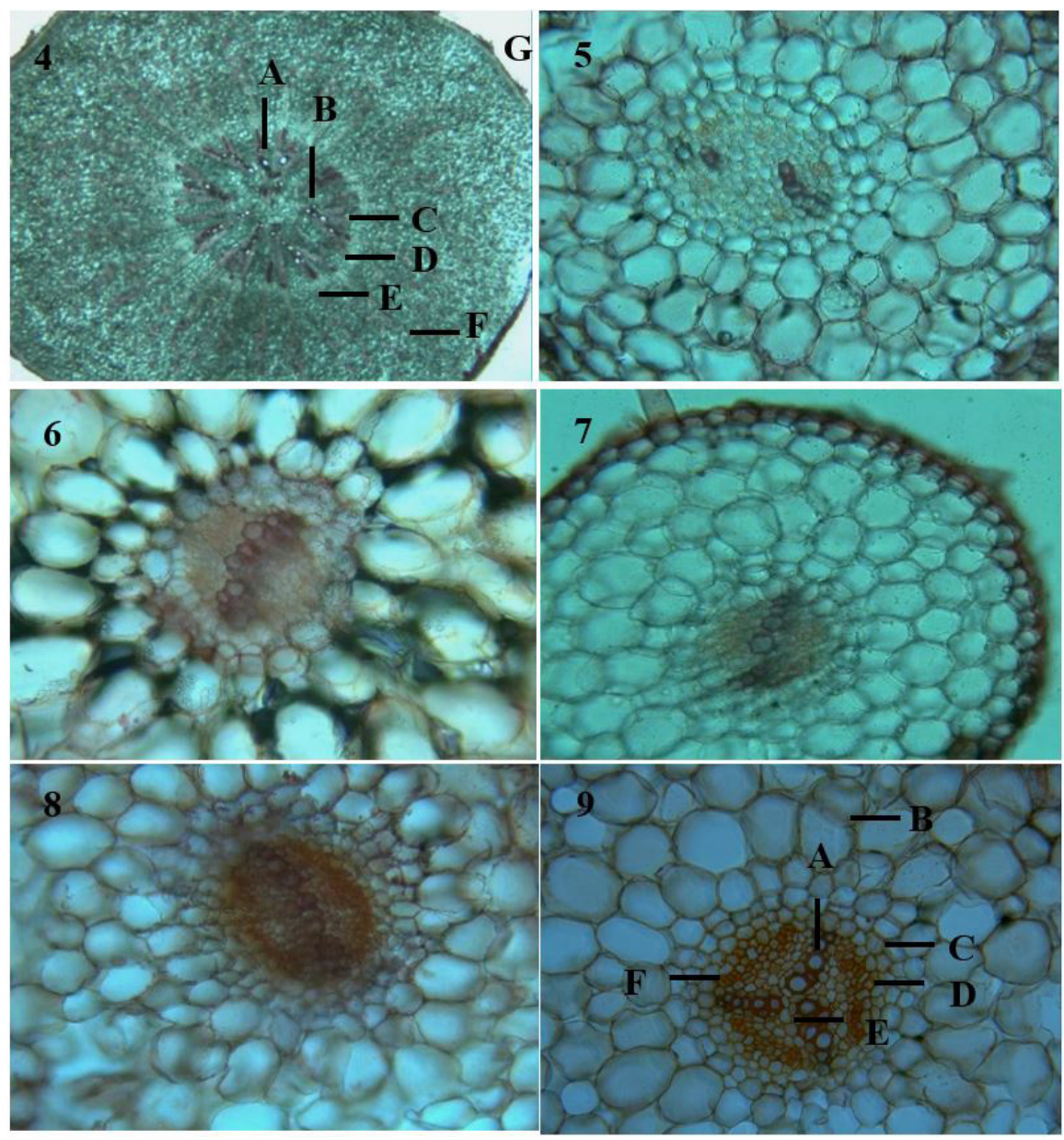

Figure.4-9: Different anatomies of roots from in vitro to ex vitro condition.

4-1-year old plantlets adapted in greenhouse.

5-2-month old in vitro rooted plantlets.

6-30-days old plantlets, dipped with IBA at $300 \mathrm{mg} / \mathrm{L}$ and transferred to 3:1:1 (sand:soil:vermiculite).

7-30-days old plantlets, dipped with IBA at $500 \mathrm{mg} / \mathrm{L}$ and transferred to 3:1:1.

8-30-days old plantlets, dipped with IBA at $300 \mathrm{mg} / \mathrm{L}$ and transferred to 5:1:1.

9-30-days old plantlets, dipped with IBA at $500 \mathrm{mg} / \mathrm{L}$ and transferred to 5:1:1.

3. (A)-Phloem; (B)-Xylem; (C)-Periderm; (D)-Pericycle; (E)-Endoderm; (F)-Bark; (G)-Epiblema 8. (A)-Xylem; (B)-Bark; (C)-Endoderm; (D)-Periderm; (E)-Phloem; (F)-Sclerenchyma 


\section{CONCLUSIONS}

1. The best multiplication of S.alopecuroides shoots were obtained on MS medium with $2.0 \mathrm{mg} / \mathrm{L}$ (IBA). The shoot average was $10.6 \pm 0.5$ that was regenerated from per explant during 4 weeks.

2. The best quality of rooted shoots was in a semi-strength MS medium supplemented with $2.0 \mathrm{mg} / \mathrm{L}$ IAA. The highest frequency of rooting was $71.5 \%$, root number per shoot was $3.8 \pm 0.4$ and the average root length was $1.2 \pm 0.1$.

3. The shoots were treated with $500 \mathrm{mg} / \mathrm{L}$
IBA and transferred in soil containing bottles with 5:1:1 (sand:soil:vermilculite) found the rooting was good forming of anatomic structure and the rooting survival rate was 80 per cent when transferred to greenhouse.

Acknowledgements: The authors wish to thank Dr. Bolortuya U. (Plant Biotechnology Laboratory, Institute of Biology, MAS) for his critical reading of the manuscript. This study was carried out with support of Dr. Tserenkhand G. head of Anatomy and Ecophysiology Laboratory.

\section{REFERENCES}

1. Cao You-Long, Li Xiao-Ying, Luo Qing, Bei Zhan-Lin (2010). Tissue culture and plant regeneration of Sophora alopecuroides. Guihaia, 30(1): pp.102 -105.

2. Dagvatseren B, Narantsetseg G, Khishigjargal L, Zina S, Oyün Z, Batchimeg U (2005). Useful manual with proper of medicinal plant: p. 174.

3. Elizabeth M. Frick and Lucia C. Strader (2018). Roles for IBA-derived auxin in plant development. Journal of Experimental Botany, 69(2): pp. 169-177.

4. Gonçalves, J. C.; Diogo, G. \& Amâncio, S (1998). In vitro propagation of chestnut (Castanea sativa $x$ C.crenata): effect of rooting treatments on plant survival, peroxidase activity and anatomical changes during adventitious root formation. Scientia Horticult., 72, pp. 265-275.

5. Iturriaga A, Jordan M, Roveraro C, Goreux A (1994). In vitro culture of Sophora toromiro (Papilionaceae), an endangered species. Plant cell tissue organ cult. 37: 2 pp. 01-204.

6. Jamyandorj, Kh., Ligaa, U., Otgonbileg, Kh, Saaral, N (2011). Rare and very rare plant cultivation in Kherlen village [in Mongolian]. p. 178.

7. Jaeyoung Kwon, Sunita Bashet, Jin Woo Lee, Eun-Kyoung Seo, Nanzad Tsevegsuren, Bang Yeon Hwang, Dongho Lee (2015). Chemical constituents isolated from the Mongolian medicinal plant Sophora alopecuroides L. and their inhibitory effects on LPSinduced nitric oxide production in RAW 264.7 macrophages. Bioorganic and Medicinal Chemistry Letters. 25: pp. 3314-3318.

8. Jordan, M., Larrain, M., Tapia, A. \& Roveraro, C (2001). In vitro regeneration of Sophora toromiro from seedling explants. Plant Cell Tissue Organ Cult.66: pp. 89-95.

9. Kartha KK, Gamborg OL (1975). Elimination of cassava mosaic disease by meristem culture. Phytopathol. 65: pp. 826-828.

10. Ligaa, U, Davaasüren, B, Ninjil, N (2005). Medicinal Plants of Mongolia Used in Western and Eastern Medicine [in Mongolian]. pp. 227-228.

11. Míriam F. Moreira, Beatriz Appezzato-da-Glória and Lilian B.P. Zaidan (2000). Anatomical aspects of IBA-treated microcuttings of Gomphrenamacrocephala St.-Hil. Brazilian Archives of Biology and Technology (2) : pp. 221-227.

12. Phulwaria M, Shekhawat NS, Rathore JS, Singh RP (2013). An efficient in vitro regeneration and ex vitro rooting of Ceropegia bulbosa Roxb.-a threatened and pharmaceutical important plant of Indian Thar Desert. Ind Crop Prod. 42: pp. 25-29. 
13. Rathore MS, Rathore MS, Shekhawat NS (2013). Ex vivo implications of phytohormones on various in vitro responses in Leptadenia reticulata (Retz.) Wight. and Arn.-an endangered plant. Environ Exp Bot. pp. 86:86-93.

14. Ranaweeraa KK, Gunasekarab MTK, Eeswara JP (2013). Ex vitro rooting: a low cost micropropagation technique for Tea (Camellia sinensis (L.) O. Kuntz) hybrids. Sci Hortic pp. 155:8-14.

15. Saito, K., Yamazaki, M., Yamakawa, K., Fujisawa, S., Takamatsu, S., Kawaguchi, A., Murakoshi, I. (1989). Lupine alkaloids in tissue culture of Sophora flavescens var. angustifolia. Greening induced production of matrine. Chern Ph arm Bull (Tokyo) pp. 37(11): 3001-3004.

16. Urgamal M, Magsar D, Sangidorj B (2003). Botany textbook: p. 252.

17. Wei Kun-Hua, Gao Shan-Lin, Huang He-Ping (2010). Tissue culture and generation of autotetraploid plants of Sophora flavescens Aiton. Pharmacognosy magazine 6(24): pp. 286- 292.

18. Zhao, D. L, Guo, G. Q, Wang, X.Y, Zheng, G. C (2003). In vitro micro propagation of medicinal plant species Sophora flavescens. Biologia plantarum. 47(1): pp. 117-120. 